\title{
Advances in Intelligent Information Processing
}

Tools and Applications 
This page intentionally left blank 


\title{
Platinum Jubilee Series
}

Statistical Science and Interdisciplinary Research - Vol. 2

\section{Advances in Intelligent Information Processing \\ Tools and Applications}

\author{
Editors \\ B. Chanda \\ C. A. Murthy \\ Indian Statistical Institute, India
}

Series Editor: Sankar K. Pal

\section{WF World Scientific}


Published by

World Scientific Publishing Co. Pte. Ltd.

5 Toh Tuck Link, Singapore 596224

USA office: 27 Warren Street, Suite 401-402, Hackensack, NJ 07601

UK office: 57 Shelton Street, Covent Garden, London WC2H 9HE

\section{British Library Cataloguing-in-Publication Data}

A catalogue record for this book is available from the British Library.

\section{ADVANCES IN INTELLIGENT INFORMATION PROCESSING: Tools and Applications \\ Statistical Science and Interdisciplinary Research - Vol. 2}

Copyright (C) 2008 by World Scientific Publishing Co. Pte. Ltd.

All rights reserved. This book, or parts thereof, may not be reproduced in any form or by any means, electronic or mechanical, including photocopying, recording or any information storage and retrieval system now known or to be invented, without written permission from the Publisher.

For photocopying of material in this volume, please pay a copying fee through the Copyright Clearance Center, Inc., 222 Rosewood Drive, Danvers, MA 01923, USA. In this case permission to photocopy is not required from the publisher.

ISBN-13 978-981-281-898-0

ISBN-10 981-281-898-7

Printed in Singapore. 


\section{Statistical Science and Interdisciplinary Research}

\section{Series Editor: Sankar K. Pal (Indian Statistical Institute)}

\section{Description:}

In conjunction with the Platinum Jubilee celebrations of the Indian Statistical Institute, a series of books will be produced to cover various topics, such as Statistics and Mathematics, Computer Science, Machine Intelligence, Econometrics, other Physical Sciences, and Social and Natural Sciences. This series of edited volumes in the mentioned disciplines culminate mostly out of significant events conferences, workshops and lectures - held at the ten branches and centers of ISI to commemorate the long history of the institute.

Vol. 1 Mathematical Programming and Game Theory for Decision Making edited by S. K. Neogy, R. B. Bapat, A. K. Das \& T. Parthasarathy (Indian Statistical Institute, India)

Vol. 2 Advances in Intelligent Information Processing: Tools and Applications edited by B. Chandra \& C. A. Murthy (Indian Statistical Institute, India) 


\section{Foreword}

The Indian Statistical Institute (ISI) was established on 17th December, 1931 by a great visionary Professor Prasanta Chandra Mahalanobis to promote research in the theory and applications of statistics as a new scientific discipline in India. In 1959, Pandit Jawaharlal Nehru, the then Prime Minister of India introduced the ISI Act in parliament and designated it as an Institution of National Importance because of its remarkable achievements in statistical work as well as its contribution to economic planning.

Today, the Indian Statistical Institute occupies a prestigious position in the academic firmament. It has been a heaven for bright and talented academics working in a number of disciplines. Its research faculty has done India proud in the arenas of Statistics, Mathematics, Economics, Computer Science, among others. Over seventy-five years, it has grown into a massive banyan tree, like the institute emblem. The Institute now serves the nation as a unified and monolithic organization from different places, namely Kolkata, the Head Quarter, Delhi and Bangalore, two centers, a network of six SQC-OR Units located at Mumbai, Pune, Baroda, Hyderabad, Chennai and Coimbatore, and a branch (field station) at Giridih.

The platinum jubilee celebrations of ISI have been launched by Honorable Prime Minister Dr. Manmohan Singh on December 24, 2006, and the Government of India has declared 29th June as the "Statistics Day" to commemorate the birthday of Professor Mahalanobis nationally.

Professor Mahalanobis, was a great believer in interdisciplinary research, because he thought that this will promote the development of not only Statistics, but also the other natural and social sciences. To promote interdisciplinary research, major strides were made in the areas of computer science, statistical quality control, economics, biological and social sciences, physical and earth sciences.

The Institute's motto of 'unity in diversity' has been the guiding principle of all its activities since its inception. It highlights the unifying role of statistics in relation to various scientific activities. 
In tune with this hallowed tradition, a comprehensive academic programme, involving Nobel Laureates, Fellows of the Royal Society, and other dignitaries, has been implemented throughout the Platinum Jubilee year, highlighting the emerging areas of ongoing frontline research in its various scientific divisions, centres, and outlying units. It includes international and national-level seminars, symposia, conferences and workshops, as well as series of special lectures. As an outcome of these events, the Institute is bringing out a series of comprehensive volumes in different subjects under the title Statistical Science and Interdisciplinary Research, published by World Scientific Publishing, Singapore.

The present volume titled Advances in Intelligent Information Processing: Tools and Applications is the second one in the series. It has thirteen chapters, written by eminent scientists from different parts of the world, dealing with different aspects, characteristics and methodologies of intelligent information processing with real life applications. Both classical and modern techniques are used. Chapters on image and video processing mainly deal with challenging problems like curve evolution for partially occluded patterns, contour tracking, region classification, object category identification in video sequence, facial expression recognition, duplicate image detection for efficient video retrieval, and hardware architecture for pixel classification. Besides, the issues of uncertainty handling in class definition by integrating fuzzy sets and rough sets with an application on bioinformatics data, and the reuse of domain knowledge in soft computing framework for efficient modeling of intelligent systems are described in two chapters. I believe the state-of-the-art studies presented in this book will be very useful to readers.

Thanks to the contributors for their excellent research contributions and to volume editors Professors Bhabatosh Chanda and C.A. Murthy for their sincere effort in bringing out the volume nicely in time. Thanks are also due to World Scientific for their initiative in publishing the series and being a part of the Platinum Jubilee endeavor of the Institute.

December 2007

Kolkata

Sankar K. Pal

Series Editor and

Director, ISI 


\section{Preface}

Intelligent information processing methodologies adopt one of the two major approaches: model based or rule based. In the first approach a model is developed from the sufficient training data, the domain knowledge as well as the knowledge of the physical process giving out the data. All the subsequent analysis and decision making is based on the model itself. Hence, though does not cover much wide spectrum, the method developed by this approach is very robust. Second approach develops a rule-base based on extensive and meticulous observation of the system and its outcome as well as reasoning. This approach covers a wide spectrum of situation, but may not be as reliable as the first one.

In the first paper, Joshi and Brady have developed a novel non-parametric mixture model for the histogram of image intensities. This leads to the evolution of level sets in a Bayesian framework, which are applied to region classification and image segmentation problem. The experimental results on medical images demonstrate the effectiveness of the method. The next paper by Chattopadhyay and Mukherjee, describes the level set based curve evolution method for pattern generation in 2D space. The reaction-diffusion and shape optimization models are used to derive constraints for curve evolution. The proposed model is extended successfully to the reconstruction of partially occluded patterns, which is a problem in Computer vision. The problem of instability in curve evolution, while using active contours for object segmentation and tracking, is tackled by Srikrishnan and Chaudhuri in the third paper. They propose the use of an additional tangential component for stability while the shape of the curve remains intact. The next article deals with object tracking, object recognition and object reconstruction using a completely different approach, i.e., general state modeling and estimation. In this work, the method proposed by Derichs, Deutsch, Wenhardt, Niemann and Denzlery here is based on determining the next best view in active state estimation using the concept of mutual information. The problem of 3D 
object recognition is tackled by Zografos and Buxton in the fifth article. Their method combines linearly a few 2-dimensional intensity images of the same $3 \mathrm{~d}$ object taken from vicinity but arbitrary viewpoints to obtain an overall idea about the object. They finally used an evolutionary algorithm for obtaining the 'optimal parameters for linear combination of views. Harit and Bharatia and Chaudhury present an approach for object category identification in video sequences. The proposed approach integrates object model knowledge with the perceptual organization process in human beings. The methods we have presented so far are for object segmentation, tracking and recognition in image or video. Emotion and expression recognition from an image is also an active research area nowadays. The article of Buciu and Pitas is on recognition of six basic facial expressions. The authors have developed a novel way of extracting features using discriminant non-negative matrix factorization algorithm. They successfully demonstrated the superior performance of their methodology over the competing strategies. Another active research area is retrieval of image and video from a large database. Search can be made more efficient and recall can be improved if the duplicate images can be trimmed off. Ghosh, Gelasca, Ramakrishnany and Manjunath tackled the problem of duplicate image detection in very large databases in the next article. They used a 12 dimensional descriptor based on Fourier Mellin transform. The detection of duplicate images is dependant o the dissimilarity measure. Another application of dissimilarity analysis may be found in the change detection problem in satellite images. Patra, Ghosh and Ghosh exploited the properties of self-organized feature map to reach a satisfactory solution to this problem. Another two important aspects of dealing with image and video data are compression and processing time. Liu, Zhu, Bosch and Delp provide an excellent review article describing the recent advances in video compression techniques. They additionally described the latest video coding standard. Compression is an essential tool for efficient storage and transmission of data. Another essential consideration is the processing time required for a given task. Hardware implementation of an algorithm always improves its efficiency in terms of speed. In the next article by Bishnu, Bhowmick, Bhattacharya, Kundu, Murthy and Acharya, a combinatorial approach is described for designing a hardware architecture for the classification each pixel into one of the three classes, namely, crest, valley and slope. The proposed pipeline design can be used to build a fast coprocessor for online finger print analysis. Intelligent information processing system should be able to handle uncertainties. Almost all the methods presented so far have some means of satisfying this criterion. For example, self-organized feature map, which is used in change detection in satellite image, has inherent capability to handle uncertainties to some extent. Rough set and fuzzy set based tools also have similar capabilities. 
Maji and Pal propose a roughfuzzy C-medoids algorithm to select most informative bio-bases for amino acid sequence analysis in bioinformatics. The superior performance of the proposed method is demonstrated on different protein datasets. As indicated in the beginning the success of an intelligent information processing system greatly depends on the proper exploitation of domain and process knowledge. In the final article, Pedrycz describes the concept of knowledge reuse in the computational intelligence models. New performance index is defined whose minimization helps in the most effective level of knowledge reuse. The utility of the proposed methodology is demonstrated on fuzzy rule based systems.

First of all we express our heart-felt gratitude towards the contributors of this volume. This volume contains extended version of some articles presented at ICCTA. So we are grateful to Organizing Committee and Programme Committee of ICCTA to allow us to use the review reports of the articles. We also like to thank the reviewers of the articles processed for this volume. We also express our gratitude to the Indian Statistical Institute Platinum Jubilee Core Committee and the Series Editor for giving us opportunity to edit this volume. Finally, the help of Mr. Dilip Gayen and Mr. Indranil Dutta to prepare the camera-ready version is gratefully acknowledged.

Bhabatosh Chanda

C. A. Murthy

Editors 
This page intentionally left blank 


\section{Contents}

Foreword $\quad$ v

Preface vii

1. Non-parametric Mixture Model Based Evolution of Level Sets 1

N. Joshi and M. Brady

$1.1 \quad$ Introduction . . . . . . . . . . . . . . . . . . 1

1.2 Need for Modelling Class Distributions Non-parametrically . . . 3

1.3 NP-windows Method for Non-parametric Estimation of PDFs . 4

1.4 NPMM-ICLS Framework . . . . . . . . . . . . . 7

1.5 Level Sets Method . . . . . . . . . . . . . . . . . 9

$1.6 \quad$ NPMM-ICLS Level Sets Method . . . . . . . . . . . . . . . 10

1.7 Results and Discussion . . . . . . . . . . . . . . . . 11

1.8 Conclusions . . . . . . . . . . . . . . . . 15

2. Pattern Generation Using Level Set Based Curve Evolution 19

A. Chattopadhyay and D. P. Mukherjee

$2.1 \quad$ Introduction . . . . . . . . . . . . . . . . . . . . 19

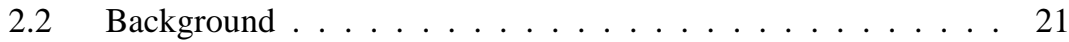

2.2.1 Level set model of curve evolution . . . . . . . . . 21

2.2.2 Reaction-diffusion model . . . . . . . . . . . 22

2.2.3 Shape optimization . . . . . . . . . . . . . 24

2.3 Proposed Methodology . . . . . . . . . . . . . . . . . . 26

2.3.1 Reaction-diffusion influenced curve evolution . . . . 26

2.3.2 Shape optimization based curve evolution . . . . . . 28 
2.4 Results . . . . . . . . . . . . . . . 30

2.4.1 Pattern disocclusion . . . . . . . . . . . . . 33

2.5 Conclusions . . . . . . . . . . . . . . 34

3. Stable Contour Tracking Through Tangential Evolution 37

V. Srikrishnan and S. Chaudhuri

3.1 Active Contours: Introduction . . . . . . . . . . . 37

3.2 Curve Evolution . . . . . . . . . . . . . . . . . . . . 39

3.3 Difficulties with Parametric Curves . . . . . . . . . . . . . . 40

3.4 Existing Solutions . . . . . . . . . . . . . . . . . . 42

3.5 Proposed Method . . . . . . . . . . . . . . . . . . 42

3.5.1 Comparison with other works . . . . . . . . . . . 44

3.5.2 Choice of the ideal constant $K \ldots \ldots \ldots$. . . . 45

3.5.3 Proof of conditional boundedness . . . . . . . . 46

3.6 Applications in Image Segmentation and Tracking . . . . . . . . 48

3.7 Implementation Details . . . . . . . . . . . . . . . . . . . . 49

3.8 Results . . . . . . . . . . . . . . . . . . . . . . . . 49

3.9 Conclusions and Future Work . . . . . . . . . . . . . 52

4. Information Theoretic Approaches for Next Best View

Planning in Active Computer Vision 55

C. Derichs, B. Deutsch, S. Wenhardt, H. Niemann and J. Denzler

4.1 Introduction . . . . . . . . . . . . . . . 56

4.2 Information Theoretical Approaches for Next Best View

Planning . . . . . . . . . . . . . . 57

4.2.1 General state modeling and estimation . . . . . . . 58

4.2.2 Optimality criteria for active view planning . . . . . . 59

4.3 Planning Tasks . . . . . . . . . . . . . . . . 62

4.3.1 Active object recognition . . . . . . . . . . . 62

4.3.2 Active object tracking . . . . . . . . . . . . . 66

4.3.3 Active object reconstruction . . . . . . . . . . . 71

4.4 Experiments . . . . . . . . . . . . . . . 74

4.4.1 Evaluation for active object recognition . . . . . . . 74

4.4.2 Evaluation for active object tracking . . . . . . . . 75

4.4.3 Evaluation for active object reconstruction . . . . . . 78

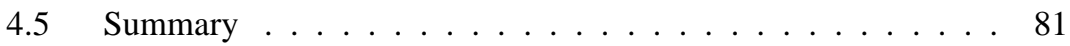


5. Evaluation of Linear Combination of Views for Object Recognition

V. Zografos and B. F. Buxton

$5.1 \quad$ Introduction . . . . . . . . . . . . . 85

5.2 Linear Combination of Views . . . . . . . . . . . . . . . 87

5.2.1 Image synthesis . . . . . . . . . . . . . . . . 88

5.3 The Recognition System ． . . . . . . . . . . . . . . . . . . . 89

5.3.1 Template matching . . . . . . . . . . . 90

5.3.2 Optimisation . . . . . . . . . . . . . . . 91

$5.4 \quad$ Experimental Results . . . . . . . . . . . . . . . . . . . . . . 94

5.4.1 Experiments on the CMU PIE database . . . . . . . . 95

5.4.2 Experiments on the COIL-20 database . . . . . . . . 99

5.5 Conclusion . . . . . . . . . . . . . . . . 103

6. Using Object Models as Domain Knowledge in Perceptual

Organization

G. Harit, R. Bharatia and S. Chaudhury

6.1 Introduction . . . . . . . . . . . . . . . . . 107

6.2 Perceptual Grouping in Video . . . . . . . . . . . . . . . . 109

6.2.1 Video data clustering . . . . . . . . . . . . . . . 109

6.2.2 The perceptual grouping model . . . . . . . . . 110

6.3 Object Model as a Pictorial Structure . . . . . . . . . . . . . . . 113

6.3.1 Formulation of the potential between object parts . . . 114

6.3.2 Formulation of appearance parameters for

object parts . . . . . . . . . . . . . . . 114

6.4 Spatio-Temporal Grouping . . . . . . . . . . . . . . . 117

6.4.1 Formulation of object model evidence . . . . . . . . 117

6.4.2 The grouping algorithm . . . . . . . . . . . 118

6.5 Results . . . . . . . . . . . . . . . . . 120

6.6 Conclusions . . . . . . . . . . . . . . . . 123

7. Image Representations Based on Discriminant Non-negative

Matrix Factorization

I. Buciu and I. Pitas

7.1 Introduction . . . . . . . . . . . . . . 126

7.2 Bregman Distance, Kullback-Leibler Divergence and

Non-negative Matrix Factorization . . . . . . . . . . . . . . 128

7.3 Local Non-negative Matrix Factorization . . . . . . . . . . . . 130 
7.4 Discriminant Non-negative Matrix Factorization . . . . . . . . 131

7.5 Facial Expression Recognition Experiment _ . . . . . . . . 133

7.5.1 Data description . . . . . . . . . . . . . . 133

7.5.2 Training procedure . . . . . . . . . . . . 134

7.5.3 Feature extraction and image representation . . . . . 134

7.5.4 Test procedure . . . . . . . . . . . . . . 139

7.5.5 Classification procedure . . . . . . . . . . . . . 139

7.6 Performance Evaluation and Discussions . . . . . . . . . . . . 139

7.7 Conclusion . . . . . . . . . . . . . . . . 143

8. Duplicate Image Detection in Large Scale Databases 149

P. Ghosh, E. D. Gelasca, K. R. Ramakrishnan and

B. S. Manjunath

8.1 Introduction . . . . . . . . . . . . . . . . . . . . 149

8.2 Related Work . . . . . . . . . . . . . . . . . . . 152

8.3 System Overview . . . . . . . . . . . . . . . . . . . . 153

8.3.1 CFMT descriptor for images . . . . . . . . . 155

8.3.2 CFMT extraction for arbitrarily shaped regions . . . . . 156

8.4 Experimental Results . . . . . . . . . . . . . . . . . . . 157

8.4.1 Performance evaluation . . . . . . . . . . . 158

8.4.2 Results on web image database . . . . . . . . . . 158

8.4 .3 Time performance . . . . . . . . . . . . . . 160

8.4.4 Results on MM270K image database . . . . . . . . . 161

8.5 Conclusion and Future Work . . . . . . . . . . . . . . . . 165

9. Unsupervised Change Detection Techniques Based on

Self-Organizing Feature Map Neural Network

S. Patra, S. Ghosh and A. Ghosh

9.1 Introduction . . . . . . . . . . . . . . . . . . 168

9.2 Kohonen's Model of Self-Organizing Feature Map _ . . . . . 170

9.3 Proposed Change Detection Techniques . . . . . . . . . . . . 171

9.3.1 Change detection based on 1D-SOFM . . . . . . 172

9.3.2 Change detection based on 2D-SOFM . . . . . . . 173

9.4 Description of the Data Sets . . . . . . . . . . . . . . . 179

9.4.1 Data set related to Mexico area . . . . . . . . . . . . 179

9.4.2 Data set related to Sardinia Island, Italy . . . . . . . 180

9.5 Experimental Results . . . . . . . . . . . . . . . . . 181

9.5.1 Description of experiments . . . . . . . . 181 
9.5.2 Results on Mexico data . . . . . . . . . . . . 183

9.5.3 Results on Sardinia Island data . . . . . . . . . . . 185

9.6 Discussion and Conclusion . . . . . . . . . . . . . . 187

10. Recent Advances in Video Compression 191

L. Liu, F. Zhu, M. Bosch, and E. J. Delp

10.1 Introduction and Overview of Video Coding Standards . . . . 191

10.2 Distributed Video Coding . . . . . . . . . . . . . . . . . . . 193

10.2.1 Wyner-Ziv video coding . . . . . . . . . . . . . 195

10.2.2 Rate distortion analysis . . . . . . . . . . . . . . . 196

10.2.3 Backward channel aware Wyner-Ziv video coding . . . 197

10.3 Texture-Based Video Coding . . . . . . . . . . . . . . . . . . 199

10.3.1 Spatial texture models . . . . . . . . . . . . . . 201

10.3.2 Temporal qnalysis . . . . . . . . . . . . . . 202

10.3.3 A new perspective on texture-based video coding . . . 203

10.4 Scalable Video Coding . . . . . . . . . . . . . . . . . . . . . . 204

10.4.1 Temporal scalability . . . . . . . . . . . . . . . 205

10.4.2 Spatial scalability . . . . . . . . . . . . . 205

10.4.3 SNR and rate scalability . . . . . . . . . . . 206

10.5 Multi-View Coding . . . . . . . . . . . . . . . 206

10.6 Conclusion . . . . . . . . . . . . . . . 208

11. Hardware Architecture for Ridge Extraction in Fingerprints 213

A. Bishnu, P. Bhowmick, J. Dey, B. B. Bhattacharya,

M. K. Kundu, C. A. Murthy, and T. Acharya

11.1 Introduction . . . . . . . . . . . . . . . . . 214

11.2 Proposed Method . . . . . . . . . . . . . . . . . . 217

11.2.1 Theme . . . . . . . . . . . . . . . . 217

11.2.2 Combinatorial possibilities . . . . . . . . . . 218

11.2.3 Implementation details . . . . . . . . . . . . 220

11.2.4 Classification of a pixel . . . . . . . . . . . . 222

11.2.5 Thinning . . . . . . . . . . . . . . 225

11.2.6 Algorithm . . . . . . . . . . . . . . . 225

11.3 Evaluation and Results . . . . . . . . . . . . . . . . . 227

11.3.1 Evaluation criteria for ridge/valley finding algorithms . . . . . . . . . . . . . . 227

11.3.2 Results . . . . . . . . . . . . . . . . . . . 228

11.4 Parallel Algorithm and Hardware Architecture . . . . . . . . . 233 
11.4.1 Parallel algorithm . . . . . . . . . . . . . . . . . . 233

11.4.2 Hardware architecture . . . . . . . . . . . . . . 233

11.4 .3 Time complexity . . . . . . . . . . . . . . . . . . . 239

11.4.4 Circuit cost . . . . . . . . . . . . . . . . . 239

11.5 Discussions and Conclusions . . . . . . . . . . . . . . 240

12. Rough-Fuzzy Hybridization for Protein Sequence Analysis 243 P. Maji and S. K. Pal

12.1 Introduction . . . . . . . . . . . . . . . . . 243

12.2 Bio-Basis Function, Rough Set, and Fuzzy Set . . . . . . . . 246

12.2.1 Bio-basis function . . . . . . . . . . . . . . . 246

12.2 .2 Rough sets . . . . . . . . . . . . . . . . . . . . 248

12.2.3 Fuzzy set . . . . . . . . . . . . . . . . . . 249

12.3 Rough-Fuzzy C-Medoids Algorithm . . . . . . . . . . . . . 249

12.3.1 Hard C-medoids . . . . . . . . . . . . . . . . . 250

12.3.2 Fuzzy C-medoids . . . . . . . . . . . . . . . 250

12.3.3 Rough C-medoids . . . . . . . . . . . . . . . 251

12.3.4 Rough-fuzzy C-medoids . . . . . . . . . . . . . . 253

12.3.5 Selection of initial bio-basis . . . . . . . . . . . 255

12.4 Quantitative Measure . . . . . . . . . . . . . . 256

12.4.1 Using homology alignment score . . . . . . . . . . 257

12.4.2 Using mutual information . . . . . . . . . . . . . 258

12.5 Experimental Results . . . . . . . . . . . . . . . . . . . 259

12.5.1 Description of data set . . . . . . . . . . . . . 259

12.5.2 Example . . . . . . . . . . . . . . . 261

12.5.3 Performance analysis . . . . . . . . . . . 262

12.6 Conclusion . . . . . . . . . . . . . . . . 273

13. Knowledge Reuse in the Design of Models of Computational

Intelligence

W. Pedrycz

13.1 Introduction . . . . . . . . . . . . . . . . . 277

13.2 Problem Formulation . . . . . . . . . . . . . . . . . 280

13.2.1 Performance index . . . . . . . . . . . . . 280

13.2.2 Optimization of the level of knowledge reuse . . . . . . 281

13.2.3 Refinement of knowledge reuse . . . . . . . . . . 283

13.3 Fuzzy Rule-based Models and Their Experience-consistent

Design . . . . . . . . . . . . . . . . . . . 284 
13.4 The Alignment of Information Granules . . . . . . . . . . 288

13.5 Granular Characterization of Experience-consistent Rule-based Models . . . . . . . . . . . . . . . . . . . . 289

13.6 Conclusions . . . . . . . . . . . . . . . . . . . . 292

$\begin{array}{ll}\text { Index } & 295\end{array}$ 